\title{
Pathophysiology of COVID-19: Why Children Fare Better than Adults?
}

\author{
Nitin Dhochak ${ }^{1} \cdot$ Tanu Singhal $^{2} \cdot$ S. K. Kabra ${ }^{1} \cdot$ Rakesh Lodha $^{1}$ \\ Received: 24 April 2020 / Accepted: 24 April 2020 / Published online: 14 May 2020 \\ (C) Dr. K C Chaudhuri Foundation 2020
}

\begin{abstract}
The world is facing Coronavirus Disease-2019 (COVID-19) pandemic, which is causing a large number of deaths and burden on intensive care facilities. It is caused by Severe Acute Respiratory Syndrome coronavirus-2 (SARS-CoV-2) originating in Wuhan, China. It has been seen that fewer children contract COVID-19 and among infected, children have less severe disease. Insights in pathophysiological mechanisms of less severity in children could be important for devising therapeutics for high-risk adults and elderly. Early closing of schools and day-care centers led to less frequent exposure and hence, lower infection rate in children. The expression of primary target receptor for SARS-CoV-2, i.e. angiotensin converting enzyme-2 (ACE-2), decreases with age. ACE2 has lung protective effects by limiting angiotensin- 2 mediated pulmonary capillary leak and inflammation. Severe COVID-19 disease is associated with high and persistent viral loads in adults. Children have strong innate immune response due to trained immunity (secondary to live-vaccines and frequent viral infections), leading to probably early control of infection at the site of entry. Adult patients show suppressed adaptive immunity and dysfunctional over-active innate immune response in severe infections, which is not seen in children. These could be related to immune-senescence in elderly. Excellent regeneration capacity of pediatric alveolar epithelium may be contributing to early recovery from COVID-19. Children, less frequently, have risk factors such as co-morbidities, smoking, and obesity. But young infants and children with pre-existing illnesses could be high risk groups and need careful monitoring. Studies describing immune-pathogenesis in COVID-19 are lacking in children and need urgent attention.
\end{abstract}

Keywords Adaptive immunity $\cdot$ ACE-2 $\cdot$ Innate immunity $\cdot$ Pathogenesis $\cdot$ SARS-CoV2

\section{Background}

Coronavirus disease 2019 (COVID-19) due to Severe Acute Respiratory Syndrome Coronavirus-2 (SARS-CoV-2) infection is the biggest public health crises of recent times [1]. As per the World Health Organization's (WHO) update dated 21 st April 2020, there have been 2,397,216 cases and 162,956 deaths worldwide [2]. SARS-CoV-2 causes acute respiratory infection with varying severity in different age groups, wherein elderly tend to have severe disease, children are relatively spared till-date [3]. Several differences in the pathogenesis of COVID-19 between children and adults have been proposed to explain these differences [4].

Rakesh Lodha

rlodha1661@gmail.com

1 Department of Pediatrics, All India Institute of Medical Sciences, New Delhi, India

2 Department of Pediatrics, Kokilaben Dhirubhai Ambani Hospital and Medical Research Institute, Mumbai, India
Insights in the protective mechanism in children could help in development of therapeutic targets for at-risk elderly population. It is equally important to identify high-risk groups in children, so that recommendations regarding therapeutics and when available, vaccines can be made. Hence, the authors conducted a narrative review focussing on the clinical manifestations, disease pathogenesis, and possible factors explaining differences between adult and pediatric infection. As COVID-19 has similarities to SARS, some findings from SARS CoV-1 pathogenesis are extrapolated to SARS-CoV-2 infection. The possibility of a change in spectrum of pediatric disease, as the pandemic evolves further, should also be kept in mind.

\section{Clinical Features and Outcomes of COVID-19 Among Adults and Children}

Among 44,762 laboratory-confirmed cases from China, only 416 (1\%) and 549 (1\%) were from age-groups $<10$ and $10-19 \mathrm{y}$, 
respectively [3]. Similarly, out of 32,437 positive laboratory tests from public health laboratories in the United States of America (USA), $168(0.5 \%)$ and 425 (1.3\%) were the $0-4$ and 5-17 y age-group, respectively. Test positivity rates among total tests were $3.9 \%$ and $6.3 \%$ in $0-4$ y and 5-17 y age group compared to overall $14.4 \%$ positivity rates [5]. Clinical features from the largest series of adults and children are summarised in Table 1. Frequency of common symptoms was lower in children compared to adults, implying more asymptomatic infections in children [6]. Median duration of fever in children was $3 \mathrm{~d}$ compared to $10 \mathrm{~d}$ in adult patients, implying shorter illness in children [7,9].

According to the Centers for Disease Control and Prevention (CDC) report, fewer children were admitted to hospital and intensive care unit (ICU) (5.7\%-20\% and $0.58 \%-2.0 \%$, respectively) than adults aged $18-64$ y (10\%$33 \%$ and $1.4 \%-4.5 \%$, respectively). But infants had higher hospitalization rates $(15 \%-62 \%)$ than older children (aged $1-17$ y) $(4.1 \%-14 \%)$ and adults [6]. There were 3 deaths $(0.1 \%)$ in children compared to overall $2.27 \%$ mortality [6]. Ages of these 3 children were not reported, but one case reported from Illinois was an infant [10]. The Chinese caseseries of 171 laboratory-confirmed children also reported one death in a 10-mo-old child, who suffered intussusception and multi-organ failure [7]. In a Chinese series of 728 laboratory-confirmed children, the proportion of 'severe and critical' cases was $8.2 \%, 2.1 \%, 0.6 \%, 1.1 \%$ and $5.1 \%$ for the age-groups of $<1,1-5,6-10,11-15$ and $>15 \mathrm{y}$, respectively [11]. These findings suggest that though overall children are less affected and have milder illness than adults, infants have more severe illness compared to older children.

\section{Is the Pattern of Age-Specific Burden of COVID-19 Unique?}

\section{Respiratory Viruses}

The Global Burden of Diseases 2016 Lower Respiratory Infections Collaborators estimated that acute lower respiratory infections (ALRI) caused 652,572 deaths [95\% uncertainty interval (UI) 586,475-720,612] in children aged $<5$ y, 1,080,958 deaths $(943,749-1,170,638)$ in adults aged $>70 \mathrm{y}$, and 2,377,697 deaths $(2,145,584$ $2,512,809$ ) in people of all ages, worldwide in the year 2016 [12]. Incidence of Respiratory Syncytial Virus (RSV) infection (per 1000 people) was about three times higher in under-5s [17 (95\% UI 10.6-26.2)], compared to adults $>70$ y $[6.3(4.9-7.8)]$. However, the estimated case fatality rate (CFR) in under-5s was approximately half of that in adults $>70 \mathrm{y}$. On the other hand, incidence
Table 1 Comparison of clinical characteristics among pediatric and adult COVID-19 case series

\begin{tabular}{|c|c|c|c|c|}
\hline \multirow{2}{*}{ Characteristics } & \multicolumn{2}{|l|}{ Pediatric } & \multicolumn{2}{|l|}{ Adult } \\
\hline & CDC report [6] & Lu X et al. 2020 [7] & CDC report [6] & Guan WJ et al. 2020 [8] \\
\hline Number of patients & $2572 *$ & 171 & $113,985^{*}$ & 1099 \\
\hline Age group, years & $<18$ & $<16$ & $18-64$ & All ages ( $99.1 \%$ above $15 \mathrm{y})$ \\
\hline Region & USA & China & USA & China \\
\hline Male & $1408(53)$ & $104(60.8)$ & $75,450(53)^{\#}$ & $637(58.1)$ \\
\hline Age, years & 11 (median) & & & $47(35,58)^{@}$ \\
\hline Fever & $163(56)$ & $71(41.5)$ & $7794(71)$ & $975(88.7)$ \\
\hline Cough & $158(54)$ & $83(48.5)$ & $8775(80)$ & $745(67.8)$ \\
\hline Fatigue & & $13(7.6)$ & & $419(38.1)$ \\
\hline Myalgia & $66(23)$ & & $6713(61)$ & $164(14.9)$ \\
\hline Headache & $81(28)$ & & $6335(58)$ & $150(13.6)$ \\
\hline Shortness of breath & $39(13)$ & $¥$ & $4674(43)$ & 205 (18.7) \\
\hline Sore throat & $71(24)$ & & $3795(35)$ & $153(13.9)$ \\
\hline Rhinorrhea & $21(7.2)$ & $13(7.6)$ & $757(6.9)$ & \\
\hline Diarrhea & $37(13)$ & $15(8.8)$ & $3353(31)$ & $42(3.8)$ \\
\hline Nausea/Vomiting & $31(11)$ & & $1746(16)$ & $55(5.0)$ \\
\hline Abdominal pain & $17(5.8)$ & & $1329(12)$ & \\
\hline
\end{tabular}

Data are summarized as number $(\%)$, unless specified. ${ }^{@}$ data summarized as median (IQR). *Denominator for estimation of symptom frequency was 291 (age < 18 y) and 10,944 (age 18-64 y), because details of symptoms were available for these patients only; ${ }^{\#}$ Includes all patients 18 y and above; ${ }^{¥} 49(28.7 \%)$ children had tachypnea on examination in hospital. $C D C$ Centers for Disease Control and Prevention 
of influenza is nearly $60 \%$ more in adults $>70$ y $[15.8$ (11.8-20.2)] as compared to under-5s [9.1 (5.4-14.8)]. Here also, the estimated CFR in adults $>70 \mathrm{y}$ is more than double of that in under-5s [12]. Other studies on RSV burden also estimate a lower incidence of RSV ALRI in adults $\geq 65 \mathrm{y}$ as compared to under-5s and a multiple-fold higher hospital CFR in adults $\geq 65$ y $[13$, 14].

Among children, the youngest children $(0-5 \mathrm{mo})$ had a higher incidence of RSV-related ALRI and higher hospitalization rates as compared to older children, but in-hospital CFR were similar in all age-groups in under-5s [14].

In the first year of the $2009 \mathrm{H} 1 \mathrm{~N} 1$ influenza pandemic in the USA, in the initial months, the number of cases were maximum in children (April 12-July 23, 2009: 0-17 y$1,580,218$ cases; $18-64$ y- 1,430,258 cases; $>65$ y- 42,292 cases); in the subsequent time-periods, the age-group 18-64 y had maximum cases (August 1-31, 2009: 0-17 y579,037 cases; $18-64$ y- 870,804 cases; $>65$ y- 155,919 cases; and September 1, 2009-April 10, 2010: 0-17 y- 17,341,749 cases; 18-64 y- 33,091,869 cases; $>65$ y- 5,745,602 cases). The older individuals $(>65 \mathrm{y}$ ) had lower attack rates as well as lower mortality, possibly due to immunity from prior exposure to H1N1 viruses [15]. It appears that on appearance of a new respiratory viral infection, children are more likely to be affected, at least initially.

\section{SARS and MERS}

SARS-CoV-1 and Middle East Respiratory Syndrome coronavirus (MERS-CoV) caused ALRI with high CFR (7-17\% for SARS-CoV-1 and 19-46\% for MERS-CoV) [16-18]. Age-wise CFR for SARS in Hong Kong was 0\%, 2\%, 10\%, $13 \%, 25 \%, 47 \%$ and $66 \%$ out of $266,418,352,260,135,147$, and 177 cases in $0-24,25-34,35-44,45-54,55-64,65-74$, and $\geq 75$ y age-group, respectively. SARS cases in age-group $0-9$ and $10-19$ y accounted for $2.9 \%$ and $5.0 \%$ of all patients, respectively [16]. Similarly, among all patients with MERS from Kingdom of Saudi Arabia between 2012 and 2015, only $32(3.4 \%)$ out of 939 patients were aged $\leq 19$ y. Odds-ratio for fatality for age-groups $\leq 19,20-39,40-59,60-79$ and $\geq 80 \mathrm{y}$ were $0.40,0.54,1,2.44$, and 6.51 , respectively [18]. There was no reported death in children due to SARS in one the series [19]. No separate data are available for MERS-related CFR for infants and young children, but fatal cases have been reported in this group [20]. These findings suggest that SARS and MERS have significantly higher CFR compared to COVID-19 but like COVID-19, children tend to be less commonly and less severely affected.

Human-to-human transmission of SARS-CoV and MERS$\mathrm{CoV}$ occurred mainly in health care settings. This is probably due to less viral shedding before symptom onset, by which time they were most probably seeking medical care [21]. It is estimated that transmission between family members occurred in only $13-21 \%$ of MERS cases and $22-39 \%$ of SARS cases [21]. The secondary attack rates reported for COVID-19 vary from 10 to $20 \%$ [22-24].

\section{Summary}

Looking at studies on various respiratory viruses, children appear to be less susceptible to infection by SARS-CoV-1, MERS-CoV, and SARS-CoV-2, as compared to other viruses such as influenza and RSV. The basic reproduction number (R0 value) represents the number of second-generation individuals that a patient can infect after entering a susceptible population under ideal conditions. The R0 values for SARSCoV-1 and MERS-CoV were estimated to be $2-5$ and $<1$, respectively [25]. There is a trend of association between higher pathogenicity and lower transmissibility. In case of influenza, while pandemic H1N1-virus binding to receptors mainly in the upper respiratory tract, caused relatively mild disease and became endemic in the population, the H7N9virus binding to receptors mainly in the lower respiratory tract, is reported to have a fatality rate of approximately $40 \%$ and has so far resulted in only a few small clusters of human-tohuman transmission [25].

Striking difference in the pandemic 2009 influenza A to COVID-19, SARS and MERS is the fact that overall number of children affected is disproportionately low in the coronavirus associated diseases. One possible explanation is that the latter were associated with high CFR and started as outbreaks, which led to strict quarantine procedures and closing of potential hotspots for pediatric infections like schools and daycare centers. As movement of children is highly limited in these times, most of the infections are spread from infected adult family members. Even within families, individuals diagnosed with SARS-CoV-2 infection are isolated, which may reduce exposure of children. Among studies of household contacts, odds of infection in children were significantly less than elderly (OR: $0.26,95 \% \mathrm{CI} 0.13-0.54$ ) and gradually increased with age $[23,24]$.

\section{Pathogenesis of COVID-19}

\section{Virology}

SARS-CoV-2 is an enveloped, positive-sense single-stranded RNA virus, from beta-coronavirus genera of coronaviridae family. The nomenclature arises from surface spike (S) proteins giving the appearance of "crown" (latin corona) on electron microscopy [1]. SARS-CoV-2 shares $88 \%$ genetic identity to two bat-derived SARS-like coronaviruses, bat-SLCoVZC45 and bat-SL-CoVZXC21, pointing towards common zoonotic origin i.e., bats. Genetic material of SARS- 
CoV-2 has about $79 \%$ and $50 \%$ sequence identity to SARSCoV-1 and MERS-CoV, respectively [26].

SARS-CoV-2 enters respiratory epithelial cells by attaching to angiotensin converting enzyme-2 (ACE-2) via S-protein; ACE-2 is also a receptor for SARS-CoV-1 [27]. Cellular entry is facilitated by proteolytic cleavage of ACE-2 by transmembrane serine protease-2 [27]. Affinity of SARSCoV-2 for ACE-2 is approximately 10-20 times higher than SARS-CoV-1, which could explain higher infectivity of SARS-CoV-2 [28]. ACE-2 is found on apical membranes of nasal, oral, nasopharyngeal and oropharyngeal mucosal epithelium, alveolar epithelium, endothelial cells of blood vessels and heart, renal tubules, and enterocytes in small intestine [29]. CD-147 (target for antibody, Meplazumab) and glucose regulated protein-78 are newly described receptors of SARSCoV-2 for cellular entry [30, 31].

\section{Clinico-Immunological Phases}

Clinico-immunological progression suggests that COVID-19 can be divided into 3 phases: (1) Flu like illness with high viral load; (2) Critical phase (decreasing viral titres with accelerated inflammatory response causing lung and end-organ injury); and (3) Recovery phase. SARS-CoV-2 titres in nasopharyngeal and endotracheal aspirate specimens are high during first week of symptoms followed by gradual decline starting at end of first week [32-35]. In nasopharyngeal swabs from 76 patients, viral loads were significantly higher in severely affected (approximately 60 times) than mildly affected patients [36]. Another study found that patients with severe illness had approximately $1 \log _{10}$ higher viral titres (not statistically significant) [33]. A small series found no difference in viral loads between mild and severe disease. But the authors analyzed plasma RNAaemia and included only 2 and 4 patients with RNAaemia in severe and mild illness, respectively [37]. After approximately 4-7 d, a proportion of patients enter critical phase which is associated with progressive decline in viral titres and worsening of inflammatory markers. Patients with more severe disease have less steep and prolonged decline in viral titres [33]. Levels of inflammatory cytokines including IL-2, IL-6, IL-7, IL-10, GCSF, IP-10, MCP-1, MIP-1A, and TNF- $\alpha$ are higher in critically ill patients than patients with mild disease $[37,38]$. Number of T-cells, helper T-cells, and memory helper T-cells are decreased and naïve helper T-cells is increased in severe group compared to mild group [39]. Increase in IgG and IgM against SARS-CoV-2 antigens [internal nucleoprotein (NP) and receptor binding protein (RBP)] was seen around day 7-10 of symptoms, followed by gradual decrease in viral load $[33,34]$. The persistent high virus titres, poorly controlled by dysfunctional immune system with elevated cytokine levels, point towards combined effect of virus mediated cytopathic effects and immune-mediated injury as the pathway of severe lung injury and multi-organ dysfunction during critical phase. Patients can succumb to illness during critical phase or gradually recover.

Choice of therapeutic agents differs in different phases of illness. Personalized approach to therapeutics by establishing state of viral loads and inflammatory profile (pro- and antiinflammatory cytokines levels) could be the best approach. Overall, anti-viral therapy in initial phase and combination of anti-viral with immune-modulators in critical phase could be an appropriate choice. As high viral titres are the most consistent feature of severe disease, more effective anti-viral drugs are the need of the hour. Early effective anti-viral therapy could possibly prevent progression of disease to critical phase and improve outcomes and intensive care burden.

There are no studies of viral loads, and cytokine levels in children with COVID-19. Immunological manifestations like lymphopenia (3.5\%) and C-reactive protein (CRP) elevation (19.7\%) are less common in children compared to adults ( $47 \%$ and $>50 \%$ respectively) [7]. Most of the children recover following initial short flu like illness without progressing to critical phase [7].

\section{Pathology of Lung Injury}

Lung specimens from early COVID-19 in two patients with concomitant lung cancer, showed proteinaceous and fibrinoid intra-alveolar exudates with mononuclear inflammation, multinucleated giant cells and type 2 pneumocyte hyperplasia [40]. Post-mortem specimen from 3 patients dying of acute respiratory failure (on day 1 , day 6 , and 2 wk of onset of respiratory failure), showed features of diffuse alveolar damage, hyaline bodies, type 2 pneumocyte hyperplasia and scanty mononuclear cells. Occasional thrombi in small pulmonary arteries and fibrotic plugs could be seen [41-43]. These findings are similar to findings of SARS-CoV-1 infection [44]. Immune staining for SARS-CoV-2 showed strong staining of alveolar epithelial cells including desquamated debris within alveoli and minimal staining of blood vessels or interstitium [42]. These findings of strong virus staining of alveolar cells with few immune cells, point more towards direct virus mediated cell injury. More post-mortem biopsies at different durations of illness, and pediatric autopsies and biopsies will provide additional information.

\section{Effect of ACE-2 Expression}

ACE-2 is a counter-regulatory enzyme of renin-angiotensinsystem which acts by converting angiotensin-2 to Ang-(1-7) form. In healthy state, ACE-2 activity maintains homeostasis between angiotensin-2 (vasoconstriction, inflammation, fibrosis and proliferation) and Ang-(1-7) pathways (vasodilatation, anti-apoptotic, anti-fibrosis, and anti-proliferation) [45]. After entering pneumocytes, SARS-CoV-2 downregulates ACE-2 
expression, decreasing angiotensin-2 metabolism. Elevated angiotensin-2 increases pulmonary vascular permeability and inflammation, hence worsening of lung injury [21, 46]. Angiotensin-2 levels have been found to be increased in COVID-19 patients compared to healthy adults [32]. Angiotensin-2 levels have significant positive correlation with SARS-CoV-2 viral loads and lung injury severity, probably via ACE-2 down-regulation [32]. Elevated angiotensin-2 levels have also been seen with respiratory syncytial virus (RSV) and avian influenza pneumonia [47, 48]. ACE-2 levels decrease in old age and co-morbidities like hypertension and diabetes, possibly explaining worse lung injury and prognosis of SARS-CoV-2 in them $[45,49]$. In a phase- 2 clinical trial of recombinant ACE-2 infusion in adults with acute respiratory distress syndrome (ARDS), there was significant decrease in angiotensin-2 levels, and increase in Ang-(1-7) and surfactant protein-4 levels [50]. High levels of ACE-2 activity in children could be protective in children with COVID-19, leading to less severe disease in this age group compared to elderly.

Hypothesis for the potential beneficial effect of ACE-2 in lung injury in COVID-19 is complicated by the fact that ACE2 is also a primary receptor for SARS-CoV-2 in lungs. While ACE-2 knockout mice showed worse lung injury with RSV and avian influenza infections, lungs of ACE-2 knockout rats had lesser virus titres, inflammatory cell infiltrates and injury score with SARS-CoV-1 infection compared to wild type rats [47, 48, 51]. Among neonates, children, adults and elderly patients with ARDS, there was no difference in ACE, ACE2 and ACE-2:ACE activity [52].

With current evidence, it is difficult to ascertain which effect of lung ACE-2 activity is more important in SARS-CoV-2 infection i.e., protection against inflammation mediated lung injury or enhanced lung injury by facilitated virus entry. As COVID-19 is more severe in infants than older children, it is to be seen whether ACE-2 enhanced viral infection is responsible for this. Recombinant ACE-2, ACE-inhibitors and angiotensin antagonists have been proposed as potential therapeutic options in adults. Studies aimed at estimation of blood angiotensin-2 levels, and ACE-2 activity in lower respiratory tract samples in children with COVID-19 are needed.

\section{Mal-Adaptive Immune Response in Elderly}

Adult patients with severe COVID-19 have delayed clearance of SARS-CoV-2 [33]. Total lymphocyte, CD4+ and CD8+ T cell, helper $\mathrm{T}$ cell and memory $\mathrm{T}$ cell counts were significantly lower in patients with severely affected patients, pointing to depressed adaptive immunity [39]. Cytokines including IL-2, IL-6, and IL-10, and blood neutrophil counts are significantly elevated in severely ill patients, representing dysfunctional and exaggerated innate response causing lung injury [38]. Innate immunity over-activity was also seen in human type
2 alveolar cell cultures infected with SARS-CoV-1, showing excessive mRNA expression of interferon- $\beta$, interferon- $\lambda$ (IL29) and other pro-inflammatory cytokines [53]. These responses point toward an uncontrolled virus-induced lung injury and secondary cytokine mediated injury. Immune system response seems to be appropriate in children, as most of the children with COVID-19 have normal lymphocyte count [7]. Studies on sequential viral loads, lymphocyte subset and alveolar lining fluid cytokine profile are lacking in children.

\section{Protective Immunological Mechanisms in Children}

\section{Trained Immunity}

Trained immunity is the functional re-programming of innate immune cells to a more activated state following initial antigen stimulation (infections or vaccination) through metabolic re-programming (enhanced Kreb's cycle) and epigenetic changes (acetylation and demethylation leading to enhanced transcription of IL- $1 \beta$, IL- 6 and TNF- $\alpha$ genes). It can affect progenitor cells of myeloid and monocyte cell lines as well as local cells (lung macrophages and dendritic cells) [54].

It has been postulated that countries with routine Bacille Calmette-Guerin (BCG) vaccination have less COVID-19 related morbidity and mortality [55]. Following BCG vaccination in adults, there is enhanced pattern recognition receptors expression in monocytes and elevation of Th1 and Th17 immune response to non-mycobacterial stimulation, up to $1 \mathrm{y}$ following vaccination [56]. In a randomized trial, BCG vaccination was associated with reduced viremia with experimental attenuated yellow virus strain infection and epigenetic changes in monocytes [57]. IL-1 $\beta$ response upregulation correlated with reduction of viremia [57]. BCG vaccination has been associated with decrease in acute upper respiratory infection (URI) in elders and decreased mortality in under-5 children [58, 59]. BCG is also emerging as an interesting option to enhance immunity of at-risk population for severe COVID-19 disease such as elderly and healthcare workers $[60,61]$. But, protective effect of BCG against infections is unlikely to last beyond 1-2 y [56]. It will be worthwhile to evaluate the role of BCG in infants and children with co-morbidities, especially in countries lacking universal BCG immunization.

Mice infected with live adenoviral vaccine vector have been shown to induce self-sustained memory alveolar macrophages [62]. Live vaccines against measles and polio have shown to improve childhood mortality, in additional to the specific infections [63]. Trained immunity due to routine live vaccines (against measles, mumps, rubella, and influenza) and frequent viral infections could be an important protective mechanism against SARS-CoV-2 infection in children. 


\section{Cross Immunity to Other Coronavirus Infections}

Cross-reactive antibodies (CR3022) targeting highly conserved domain of SARS-CoV-1 and SARS-CoV-2 showed low affinity for SARS-CoV-2 [64]. Antibodies against conserved epitopes of influenza following influenza infection have been shown to have heterosubtypic influenza immunity $[65,66]$. As coronavirus infections are common causes of URI in children, protective role of antibodies from other coronavirus infections against SARS-CoV-2 cannot be ruled out. There are also concerns of antibody-dependent enhancement of disease severity due to low affinity antibodies from other coronavirus infections including SARS, which need considerations while developing vaccines and administering convalescent plasma in severe SARS-CoV-19 [67]. Till now, there are no reports of COVID-19 in patients who suffered from SARS or MERS previously.

\section{Developmental Changes in Immunity}

Antigen naïve immature immune system in young infants and ageing related relative immune deficiency/ dysfunction in elderly, predisposes these populations to severe ALRI to viruses like RSV and seasonal influenza [68]. As highlighted above, still younger children fare better than the elderly in most scenarios.

Predominant response to infectious stimulus in young infants is innate response and less prominent adaptive response. In a study from Latin America of children from age groups 69, 22-26, and 48-60 mo, younger children had higher release of cytokines IL-6, IL-8, IL-10 and TNF-alpha to toll likereceptor (TLR) stimulation i.e., more robust innate response. The percentage of CD4+ naïve T-cells decreased while memory $\mathrm{T}$ cells increased with age [69]. An inadequate adaptive response could explain worse course of illness seen in COVID-19 affected young infants compared to older children. Also, maternal antibodies protecting neonates and young infants, are unlikely to act against novel viruses like SARSCoV-2 [70].

Ageing related immune-senescence in the elderly predisposes them to increased incidence and severity of ALRIs. RSV infection in aged cotton rats showed prolonged viral replication and delayed rise in cytokine levels compared to younger rats [71]. Multiple innate immunity aberrations have been reported in elderly: reduced dendritic cell number and function, decreased TLR induction, upregulation of proinflammatory cytokines, decreased macrophage and neutrophil function, reduced NK-cell activity, decreased $\gamma \delta \mathrm{T}$ cell proliferation and number [72,73]. These factors lead to excessively pro-inflammatory response to infections; as well as delay in or lack of switch from an innate immune response to an adaptive immune response [21]. In patients with SARS, the survivors had a gene expression profile indicative of development of an adaptive immune response while deceased showed persistently elevated levels of pro-inflammatory cytokines encoded by the interferon-stimulated genes [74].

\section{Effects of Lung Development}

While ageing, lungs gradually have impaired regenerative potential [75]. In a study of young (2-3 mo) and aged (16-18 mo) mice, influenza virus infection led to more severe alveolar damage, and delayed regeneration of alveolar type II cells and pro-SPC-positive bronchiolar epithelial cells in older mice [76]. Good regenerative capacity could explain overall less severity and early recovery of COVID-19 in children compared to older patients.

It will be interesting to see if there is any difference in affinity of SARS-CoV-2 to different parts of respiratory epithelium with age and if pediatric infections are limited to upper respiratory tract? Due to greater upper airway resistance in children, aerosol particles deposit more in tracheobronchial tree than alveoli [77]. This could lead to more bronchiolitislike infections and less pneumonia with SARS-CoV-2 infection in children.

\section{Co-morbid Conditions}

In adults with COVID-19, co-morbidities like heart diseases, cerebrovascular diseases, and obesity are associated with increased mortality [78, 79]. Upregulation of ACE-2 in smokers may contribute to poor outcomes of respiratory infections in them; the same will be applicable to elderly smokers [80, 81]. As most of these morbidities are not commonly seen in children, children are placed in a favourable position. Secondhand smoking could be a risk factor for children.

In a study of infants with RSV ALRI, presence of prematurity, co-existing heart disease, and recurrent wheezing were associated with increased mortality [82]. Similarly, among children dying of influenza infection in USA, 57\% had atleast one high risk condition, including neurological disorders (33\%), pulmonary disorders (26\%) (including asthma, 16\%), genetic disorders (12\%) and cardiac diseases (11\%) [83]. From USA COVID-19 data, of the 3 pediatric deaths, all had associated underlying conditions [84]. Pending detailed reports, young infants and children with similar comorbidities should be considered as high-risk population for COVID-19. There is a need to study risk factors for severe SARS-CoV-2 infection in children, to identify population most appropriate for clinical trials and administration of antiviral therapies and vaccines (once available). 
Table 2 Potential factors protecting children against severe SARS-CoV-2 infection

\begin{tabular}{ll}
\hline Potential protective factor & Mechanisms \\
\hline Prevention of virus exposure & Early isolation and movement restriction \\
& -Closing schools and day-care centers in epidemics \\
Appropriate infection handling & Trained immunity (strong innate response) due to \\
& -Live vaccines (BCG, live virus vaccines) \\
& -Frequent virus infections \\
& High ACE-2 expression metabolizing angiotensin-2 \\
& Lack of immune-senescence \\
& Good lung regeneration capacity \\
& Absence of ageing related co-morbidities. \\
Absence of high-risk factors & Less degree of obesity, smoking \\
& \\
High-risk group & \\
1. Infants (<1 y) & \\
2. Children with pre-existing illnesses (neurological disorders, chronic lung diseases including asthma, un- \\
corrected heart diseases, and genetic disorders)
\end{tabular}

$A C E-2$ Angiotensin converting enzyme -2; $B C G$ Bacille Calmette-Guerin

\section{Other Mechanisms}

There are increasing reports of thrombotic complications with severe COVID-19 [85, 86]. With presence of ACE-2 on vascular endothelium and association of severe disease with raised D-dimer levels, potential role of endotheliopathy and microangiopathy in COVID-19 pathogenesis needs evaluation [78]. There are no such reports in children yet. There had been cases where patients initially turned negative for SARS-CoV-2, returned positive (not reported in children yet) [87]. But these patients were asymptomatic and likely represent carrier state than re-infection. Duration of longterm immunity is yet to be established for the novel virus.

Summary of potential factors protecting children against SARS-CoV-19 infection are summarized in Table 2.

\section{Future Directions}

1. For better definition of disease course, trends of viral loads and immune response (blood and alveolar fluid cell immune-phenotyping and cytokine levels) should be evaluated in detail in children.

2. Facilitation and description of pediatric autopsies and post-mortem lung biopsies is needed for defining patterns of lung injury.

3. As high viral titres and persistent high viral loads appear to be consistent with severe COVID-19, development of potent anti-viral agents should be expedited.

4. Evolution of ACE-2 expression and other potential receptors for the virus in different age groups and different sites in respiratory system in children needs evaluation.

\section{Conclusions}

COVID-19 is posing tremendous challenges to the entire world. Till date, children have been relatively spared. Possible mechanisms involve differences in ACE-2 expression, innate immunity, trained immunity and effects of the containment strategies including closure of schools and daycare centers.

Authors' Contributions ND: Involved in literature search, and manuscript writing; TS, SKK: Involved in literature search and review of manuscript; RL: Involved in literature search and manuscript writing and will act as guarantor for the paper.

\section{Compliance with Ethical Standards}

Conflict of Interest None.

\section{References}

1. Jin Y, Yang H, Ji W, et al. Virology, epidemiology, pathogenesis, and control of COVID-19. Viruses. 2020;12:e372.

2. WHO COVID-19 Dashboard. Available at: https://covid19.who. int/. Accessed 21 Apr 2020.

3. Wu Z, McGoogan JM. Characteristics of and important lessons from the coronavirus disease 2019 (COVID-19) outbreak in China: summary of a report of 72314 cases from the Chinese Center for Disease Control and Prevention. JAMA. 2020;323: 1239. https://doi.org/10.1001/jama.2020.2648.

4. Lee P-I, Hu Y-L, Chen P-Y, Huang Y-C, Hsueh P-R. Are children less susceptible to COVID-19? J Microbiol Immunol Infect. 2020. https://doi.org/10.1016/j.jmii.2020.02.011.

5. Coronavirus Disease 2019 (COVID-19). Available at: https://www. cdc.gov/coronavirus/2019-ncov/covid-data/covidview/04102020/ labs-regions.html. Accessed 14 April 2020. 
6. CDC COVID-19 Response Team. Coronavirus disease 2019 in children - United States, February 12-April 2, 2020. MMWR Morb Mortal Wkly Rep. 2020; https://doi.org/10.15585/mmwr. mm6914e4.

7. Lu X, Zhang L, Du H, et al. SARS-CoV-2 infection in children. $\mathrm{N}$ Engl J Med. 2020;382:1663-5. https://doi.org/10.1056/ NEJMc2005073.

8. Guan W-J, Ni Z-Y, Hu Y, et al. Clinical characteristics of coronavirus disease 2019 in China. N Engl J Med. 2020. https://doi.org/10. 1056/NEJMoa2002032.

9. Chen J, Qi T, Liu L, et al. Clinical progression of patients with COVID-19 in Shanghai, China. J Infect. 2020;80:e1-6.

10. First US Infant Death Linked to COVID-19 reported in Illinois. Available at: https://www.livescience.com/us-infant-diescoronavirus.html. Accessed 17 April 2020.

11. Dong Y, Mo X, Hu Y, et al. Epidemiology of COVID-19 among children in China. Pediatrics. 2020:e20200702. https://doi.org/10. 1542/peds.2020-0702

12. GBD 2016 Lower Respiratory Infections Collaborators. Estimates of the global, regional, and national morbidity, mortality, and aetiologies of lower respiratory infections in 195 countries, 1990-2016: A systematic analysis for the Global Burden of Disease Study 2016. Lancet Infect Dis. 2018;18:1191-210.

13. Shi T, Denouel A, Tietjen AK, et al. Global disease burden estimates of respiratory syncytial virus-associated acute respiratory infection in older adults in 2015: a systematic review and meta-analysis. J Infect Dis. 2019. https://doi.org/10.1093/infdis/jiz059.

14. Shi T, McAllister DA, O'Brien KL, et al. Global, regional, and national disease burden estimates of acute lower respiratory infections due to respiratory syncytial virus in young children in 2015: a systematic review and modelling study. Lancet. 2017;390:946-58.

15. Shrestha SS, Swerdlow DL, Borse RH, et al. Estimating the burden of 2009 pandemic influenza A (H1N1) in the United States (April 2009-April 2010). Clin Infect Dis. 2011;52:s75-82.

16. Yu PLH, Chan JSK, Fung WK. Statistical exploration from SARS. Am Stat. 2006;60:81-91.

17. Rahman A, Sarkar A. Risk factors for fatal Middle East respiratory syndrome coronavirus infections in Saudi Arabia: analysis of the WHO line list, 2013-2018. Am J Public Health. 2019;109:1288-93.

18. Alsahafi AJ, Cheng AC. The epidemiology of Middle East respiratory syndrome coronavirus in the Kingdom of Saudi Arabia, 20122015. Int J Infect Dis. 2016;45:1-4.

19. Li AM, Ng PC. Severe acute respiratory syndrome (SARS) in neonates and children. Arch Dis Child Fetal Neonatal Ed. 2005;90: f461-5.

20. Thabet F, Chehab M, Bafaqih H, Al Mohaimeed S. Middle East respiratory syndrome coronavirus in children. Saudi Med J. 2015;36:484-6.

21. de Wit E, van Doremalen N, Falzarano D, Munster VJ. SARS and MERS: recent insights into emerging coronaviruses. Nat Rev Microbiol. 2016;14:523-34.

22. Bi Q, Wu Y, Mei S, et al. Epidemiology and transmission of COVID-19 in Shenzhen China: Analysis of 391 cases and 1,286 of their close contacts. medRxiv. 2020; https://doi.org/10.1101/ 2020.03.03.20028423.

23. Luo L, Liu D, Liao X, et al. Modes of contact and risk of transmission in COVID-19 among close contacts. medRxiv. 2020; https:// doi.org/10.1101/2020.03.24.20042606.

24. Jing Q-L, Liu M-J, Yuan J, et al. Household secondary attack rate of COVID-19 and associated determinants. medRxiv. 2020; https:// doi.org/10.1101/2020.04.11.20056010.

25. Chen J. Pathogenicity and transmissibility of 2019-nCoV-A quick overview and comparison with other emerging viruses. Microbes Infect. 2020;22:69-71.
26. Lu R, Zhao X, Li J, et al. Genomic characterisation and epidemiology of 2019 novel coronavirus: implications for virus origins and receptor binding. Lancet. 2020;395:565-74.

27. Hoffmann M, Kleine-Weber H, Schroeder S, et al. SARS-CoV-2 cell entry depends on ACE2 and TMPRSS2 and is blocked by a clinically proven protease inhibitor. Cell. 2020;181:271-80.

28. Wrapp D, Wang N, Corbett KS, et al. Cryo-EM structure of the 2019-nCoV spike in the prefusion conformation. Science. 2020;367:1260-3.

29. Song R, Preston G, Yosypiv IV. Ontogeny of angiotensinconverting enzyme 2. Pediatr Res. 2012;71:13-9.

30. Wang K, Chen W, Zhou Y-S, et al. SARS-CoV-2 invades host cells via a novel route: CD147-spike protein. bioRxiv. 2020;https://doi. org/10.1101/2020.03.14.988345.

31. Ibrahim IM, Abdelmalek DH, Elshahat ME, Elfiky AA. COVID-19 spike-host cell receptor GRP78 binding site prediction. J Infect. 2020;80:554-62.

32. Liu Y, Yang Y, Zhang C, et al. Clinical and biochemical indexes from 2019-nCoV infected patients linked to viral loads and lung injury. Sci China Life Sci. 2020;63:364-74.

33. To KK-W, Tsang OT-Y, Leung W-S, et al. Temporal profiles of viral load in posterior oropharyngeal saliva samples and serum antibody responses during infection by SARS-CoV-2: an observational cohort study. Lancet Infect Dis. 2020. https://doi.org/10.1016/S14733099(20)30196-1.

34. Wölfel R, Corman VM, Guggemos W, et al. Virological assessment of hospitalized patients with COVID-2019. Nature. 2020. https:// doi.org/10.1038/s41586-020-2196-x.

35. Zou L, Ruan F, Huang M, et al. SARS-CoV-2 viral load in upper respiratory specimens of infected patients. N Engl J Med. 2020;382: 1177-9.

36. Liu Y, Yan L-M, Wan L, et al. Viral dynamics in mild and severe cases of COVID-19. Lancet Infect Dis. 2020. https://doi.org/10. 1016/S1473-3099(20)30232-2.

37. Huang C, Wang Y, Li X, et al. Clinical features of patients infected with 2019 novel coronavirus in Wuhan, China. Lancet. 2020;395: 497-506.

38. Shi Y, Tan M, Chen X, et al. Immunopathological characteristics of coronavirus disease 2019 cases in Guangzhou, China. medRxiv. 2020; https://doi.org/10.1101/2020.03.12.20034736

39. Qin C, Zhou L, Hu Z, et al. Dysregulation of immune response in patients with COVID-19 in Wuhan, China. Clin Infect Dis. 2020; https://doi.org/10.1093/cid/ciaa248/5803306.

40. Tian S, Hu W, Niu L, Liu H, Xu H, Xiao S-Y. Pulmonary pathology of early-phase 2019 novel coronavirus (COVID-19) pneumonia in two patients with lung cancer. J Thorac Oncol. 2020;15:700-4. https://doi.org/10.1016/j.jtho.2020.02.010.

41. Xu Z, Shi L, Wang Y, et al. Pathological findings of COVID-19 associated with acute respiratory distress syndrome. Lancet Respir Med. 2020;8:420-2.

42. Zhang $\mathrm{H}$, Zhou P, Wei $\mathrm{Y}$, et al. Histopathologic changes and SARSCoV-2 immunostaining in the lung of a patient with COVID-19. Ann Intern Med. 2020. https://doi.org/10.7326/M20-0533.

43. Barton LM, Duval EJ, Stroberg E, Ghosh S, Mukhopadhyay S. COVID-19 autopsies, Oklahoma, USA. Am J Clin Pathol. 2020. https://doi.org/10.1093/ajcp/aqaa062/5818922.

44. Hwang DM, Chamberlain DW, Poutanen SM, Low DE, Asa SL, Butany J. Pulmonary pathology of severe acute respiratory syndrome in Toronto. Mod Pathol. 2005;18:1-10.

45. Junyi G, Zheng H, Li L, Jiagao L. Coronavirus disease 2019 (COVID-19) and cardiovascular disease: a viewpoint on the potential influence of angiotensin-converting enzyme inhibitors/ angiotensin receptor blockers on onset and severity of severe acute respiratory syndrome coronavirus 2 infection. J Am Heart Assoc. 2020;9:e016219. 
46. Imai $\mathrm{Y}, \mathrm{Kuba} \mathrm{K}, \mathrm{Rao} \mathrm{S}$, et al. Angiotensin-converting enzyme 2 protects from severe acute lung failure. Nature. 2005;436:112-6.

47. $\mathrm{Gu} \mathrm{H}, \mathrm{Xie} \mathrm{Z}, \mathrm{Li} \mathrm{T}$, et al. Angiotensin-converting enzyme 2 inhibits lung injury induced by respiratory syncytial virus. Sci Rep. 2016;6: 19840.

48. Zou Z, Yan Y, Shu Y, et al. Angiotensin-converting enzyme 2 protects from lethal avian influenza A H5N1 infections. Nat Commun. 2014;5:3594

49. Xie X, Xudong X, Chen J, et al. Age- and gender-related difference of ACE2 expression in rat lung. Life Sci. 2006;78:2166-71.

50. Khan A, Benthin C, Zeno B, et al. A pilot clinical trial of recombinant human angiotensin-converting enzyme 2 in acute respiratory distress syndrome. Crit Care. 2017;21:234.

51. Kuba K, Imai Y, Rao S, et al. A crucial role of angiotensin converting enzyme 2 (ACE2) in SARS coronavirus-induced lung injury. Nat Med. 2005;11:875-9.

52. Schouten LR, van Kaam AH, Kohse F, et al. Age-dependent differences in pulmonary host responses in ARDS: a prospective observational cohort study. Ann Intensive Care. 2019;9:55.

53. Qian Z, Travanty EA, Oko L, et al. Innate immune response of human alveolar type II cells infected with severe acute respiratory syndrome-coronavirus. Am J Respir Cell Mol Biol. 2013;48:7428 .

54. Netea MG, Domínguez-Andrés J, Barreiro LB, et al. Defining trained immunity and its role in health and disease. Nat Rev Immunol. 2020. https://doi.org/10.1038/s41577-020-0285-6.

55. Miller A, Reandelar MJ, Fasciglione K, Roumenova V, Li Y, Otazu GH. Correlation between universal BCG vaccination policy and reduced morbidity and mortality for COVID-19: An epidemiological study. medRxiv. 2020; https://doi.org/10.1101/2020.03.24. 20042937.

56. Kleinnijenhuis J, Quintin J, Preijers F, et al. Long-lasting effects of BCG vaccination on both heterologous Th1/Th17 responses and innate trained immunity. J Innate Immun. 2014;6:152-8.

57. Arts RJW, Moorlag SJCFM, Novakovic B, et al. BCG vaccination protects against experimental viral infection in humans through the induction of cytokines associated with trained immunity. Cell Host Microbe. 2018:23:89-100.

58. Wardhana, Datau EA, Sultana A, Mandang VVV, Jim E. The efficacy of Bacillus Calmette-Guerin vaccinations for the prevention of acute upper respiratory tract infection in the elderly. Acta Med Indones. 2011;43:185-90.

59. Nankabirwa V, Tumwine JK, Mugaba PM, Tylleskär T, Sommerfelt H, PROMISE- EBF Study Group. Child survival and BCG vaccination: A community based prospective cohort study in Uganda. BMC Public Health. 2015;15:175.

60. BCG Vaccination to Protect Healthcare Workers against COVID19. Available at: https://clinicaltrials.gov/ct2/show/NCT04327206. Accessed 12 April 2020.

61. Reducing Health Care Workers Absenteeism in COVID-19 Pandemic through BCG Vaccine. Available at: https:// clinicaltrials.gov/ct2/show/NCT04328441. Accessed 12 April 2020.

62. Yao Y, Jeyanathan M, Haddadi S, et al. Induction of autonomous memory alveolar macrophages requires $\mathrm{T}$ cell help and is critical to trained immunity. Cell. 2018;175:1634-50.

63. Sankoh O, Welaga P, Debpuur C, et al. The non-specific effects of vaccines and other childhood interventions: the contribution of INDEPTH health and demographic surveillance systems. Int $\mathbf{J}$ Epidemiol. 2014;43:645-53.

64. Yuan $\mathrm{M}, \mathrm{Wu} \mathrm{NC}, \mathrm{Zhu} \mathrm{X}$, et al. A highly conserved cryptic epitope in the receptor-binding domains of SARS-CoV-2 and SARS-CoV. Science. 2020:eabb7269. https://doi.org/10.1126/science.abb7269.
65. Baumgarth N. How specific is too specific? B-cell responses to viral infections reveal the importance of breadth over depth. Immunol Rev. 2013;255:82-94.

66. Braciale TJ, Hahn YS. Immunity to viruses. Immunol Rev. 2013;255:5-12.

67. Shen C, Wang Z, Zhao F, et al. Treatment of 5 critically ill patients with COVID-19 with convalescent plasma. JAMA. 2020. https:// doi.org/10.1001/jama.2020.4783.

68. Simon AK, Hollander GA, McMichael A. Evolution of the immune system in humans from infancy to old age. Proc Biol Sci. 2015;282: 20143085.

69. Teran R, Mitre E, Vaca M, et al. Immune system development during early childhood in tropical Latin America: evidence for the age-dependent down regulation of the innate immune response. Clin Immunol. 2011;138:299-310.

70. Pou C, Nkulikiyimfura D, Henckel E, et al. The repertoire of maternal anti-viral antibodies in human newborns. Nat Med. 2019;25: 591-6.

71. Boukhvalova MS, Yim KC, Kuhn KH, et al. Age-related differences in pulmonary cytokine response to respiratory syncytial virus infection: modulation by anti-inflammatory and antiviral treatment. J Infect Dis. 2007;195:511-8.

72. Meyer KC. The role of immunity and inflammation in lung senescence and susceptibility to infection in the elderly. Semin Respir Crit Care Med. 2010;31:561-74.

73. Lowery EM, Brubaker AL, Kuhlmann E, Kovacs EJ. The aging lung. Clin Interv Aging. 2013;8:1489-96.

74. Cameron MJ, Ran L, Xu L, et al. Interferon-mediated immunopathological events are associated with atypical innate and adaptive immune responses in patients with severe acute respiratory syndrome. J Virol. 2007;81:8692-706.

75. Navarro S, Driscoll B. Regeneration of the aging lung: a mini-review. Gerontology. 2017;63:270-80

76. Yin L, Zheng D, Limmon GV, et al. Aging exacerbates damage and delays repair of alveolar epithelia following influenza viral pneumonia. Respir Res. 2014;15:116.

77. Phalen RF, Oldham MJ, Beaucage CB, Crocker TT, Mortensen JD. Postnatal enlargement of human tracheobronchial airways and implications for particle deposition. Anat Rec. 1985;212:368-80.

78. Petrilli CM, Jones SA, Yang J, et al. Factors associated with hospitalization and critical illness among 4,103 patients with COVID-19 disease in New York City. medRxiv. 2020; https://oi.org/10.1101/ 2020.04.08.20057794.

79. Du R-H, Liang L-R, Yang C-Q, et al. Predictors of mortality for patients with COVID-19 pneumonia caused by SARS-CoV-2: a prospective cohort study. Eur Respir J. 2020:2000524. https://doi. org/10.1183/13993003.00524-2020.

80. Brake SJ, Barnsley K, Lu W, McAlinden KD, Eapen MS, Sohal SS. Smoking upregulates angiotensin-converting enzyme-2 receptor: a potential adhesion site for novel coronavirus SARS-CoV-2 (Covid19). J Clin Med. 2020;9:841.

81. Vardavas CI, Nikitara K. COVID-19 and smoking: a systematic review of the evidence. Tob Induc Dis. 2020;18:20.

82. Geoghegan S, Erviti A, Caballero MT, et al. Mortality due to respiratory syncytial virus. Burden and risk factors. Am J Respir Crit Care Med. 2016;195:96-103.

83. Wong KK, Jain S, Blanton L, et al. Influenza-associated pediatric deaths in the United States, 2004-2012. Pediatrics. 2013;132:796804.

84. Coronavirus Disease 2019 (COVID-19) Daily Data Summary. Available at: https://www1.nyc.gov/assets/doh/downloads/pdf/ $\mathrm{imm} /$ covid-19-daily-data-summary-deaths-04152020-1.pdf. Accessed 17 April 2020. 
85. Davoodi L, Taghavi M, Razavi A. COVID-19 presented with deep vein thrombosis: An unusual case report. Res Square. 2020; https:// doi.org/10.21203/rs.3.rs-21602/v1.

86. Rotzinger DC, Beigelman-Aubry C, von Garnier C, Qanadli SD. Pulmonary embolism in patients with COVID-19: time to change the paradigm of computed tomography. Thromb Res. 2020;190: $58-9$.
87. Lan L, Xu D, Ye G, et al. Positive RT-PCR test results in patients recovered from COVID-19. JAMA. 2020. https://doi.org/10.1001/ jama.2020.2783.

Publisher's Note Springer Nature remains neutral with regard to jurisdictional claims in published maps and institutional affiliations. 